Revista Signos

2010, 43(72)

$71-98$

\title{
Transitividad, modo y tema en español: Un primer análisis en términos de la gramática de Cardiff
}

\author{
José María Gil \\ Adolfo García \\ Universidad Nacional de Mar del Plata-CONICET \\ Argentina
}

Resumen: Para la Teoría Sistémico-Funcional, la estructura del sistema lingüístico tiene características particulares debido a las funciones que los hablantes utilizan del lenguaje. Existen, desde luego, importantes diferencias con respecto a los usos concretos que hacen los hablantes de diferentes comunidades. Por ejemplo, no todos los grupos humanos planifican y efectúan excursiones de pesca costera en lanchitas amarillas. Sin embargo, en todas las comunidades humanas el lenguaje se usa para organizar la experiencia, para establecer vínculos sociales con los demás y para producir textos (entre varios otros fines). Los tres grandes usos recién mencionados se definen como las funciones 'experiencial', 'interpersonal' y 'textual' (y más que simples funciones son 'metafunciones', dado que forman parte del sistema lingüístico y, con ello, del potencial de significado). En este contexto, Fawcett (2008) destaca el 'principio multifuncional' del lenguaje enunciado por Halliday (1967a, 1967b): Toda cláusula desempeña varias funciones al mismo tiempo. Intentaremos mostrar que, a partir de la metodología desarrollada por la Gramática de Cardiff, puede explicarse 'cómo' las metafunciones experiencial, interpersonal y textual están presentes en la estructura de la cláusula castellana.

Palabras Claves: Sistémico-funcional, Cardiff, multifuncional, cláusula, español.

Recibido: 25-VI-2008 Aceptado: 9-VII-2009
Correspondencia: José María Gil (josemaria@gilmdq.com). Universidad Nacional de Mar del Plata. Alberti 190, 7600 Mar del Plata, Argentina. 


\section{Transitivity, mood and theme in Spanish: A first analysis in terms of Cardiff Grammar}

Abstract: Systemic-Functional Linguistics posits that the linguistic system is structured as it is because of the functions people have it serve. Needless to say, speakers from different communities do not use language for the same specific ends. For instance, not every human group plans and goes on fishing excursions on yellow boats. However, all human communities use language to organize their experience, to establish and maintain social bonds with the others, and to produce texts (to name but a few purposes). These three uses are respectively defined as the 'experiential', 'interpersonal' and 'textual' functions (or, more precisely, 'metafunctions'). In this context, Fawcett (2008) emphasizes the 'multifunctional principle' of language proposed by Halliday (1967a, 1967b): Every clause fulfills several functions at the same time. This paper will try to prove that the Cardiff Grammar provides a framework by reference to which it is possible to explain 'how' the experiential, interpersonal and textual metafunctions manifest themselves in the structure of the Spanish clause.

Key Words: Systemic-Functional Grammar, Cardiff, multifunctional, clause, Spanish.

\section{INTRODUCCIÓN}

Desde una perspectiva sistémico-funcional, resulta plausible la hipótesis de que la cláusula castellana es una estructura sintáctica que expresa varios significados al mismo tiempo. De manera concreta, puede sugerirse que los significados experiencial, interpersonal y textual son parte constitutiva de la cláusula española. Sobre la base de esta hipótesis podrá entenderse la estructura y el significado de la cláusula castellana. Para alcanzar este objetivo se emplearán conceptos y métodos de la versión de la Gramática Sistémico-Funcional que ha llegado a conocerse como la Gramática de Cardiff (Fawcett, 2008). Una buena parte de este 'dialecto funcionalista' aparece en Fawcett (2000a), donde existen definiciones de todas las unidades principales. Tucker (1998) hace un estudio exhaustivo de las 'cualidades' (adjetivos y adverbios de modo) y de su realización en el 'grupo de cualidad'. Huang $(1996,2002)$ efectúa una descripción abarcadora de la 'construcción experiencial de tema ampliado'. El mismo Fawcett (1999) describe en detalle la posición de la Gramática de Cardiff sobre los tipos principales de cláusulas subordinadas, mientras que Ball y Tucker (2004) brindan una lista de los variados y funcionalmente distintos 'Adjuntos' experienciales. Tench (1996) se dedica a la entonación inglesa. En relación con un tema que se tratará especialmente en el inciso 3 de este trabajo, Fawcett (1999) establece la posición de la Gramática de Cardiff sobre el concepto de Sujeto en inglés y el mismo Fawcett (2000b, 2000c) muestra las ventajas de un modelo sintáctico en el cual no existe el 'grupo verbal' en términos de Halliday $(1985,1994)$ y Halliday y Matthiessen (2004). Fawcett (2007a) describe la estructura de 'selección’ dentro del grupo nominal; Faw- 
cett (2007b) también caracteriza muchos de los tipos de Extensión de Auxiliar que se necesitan para una gramática completa de la cláusula inglesa.

Aun cuando no es un lingüista sistémico-funcionalista, Butler (2003a, 2003b) analiza las corrientes 'estructurales-funcionales' del lenguaje que él considera más valiosas y en su 'evaluación final' escribe que "no hay ninguna duda de que la gramática sistémico-funcional ha sobrevivido a su propio manifiesto de ser una teoría del lenguaje orientada al texto" y que "ha logrado una cobertura de la gramática del inglés mucho más amplia que otros enfoques, lo cual es especialmente verdadero para el caso de la Gramática de Cardiff” (Butler, 2003b: 471).

En lo que respecta al estudio del español, el mismo Fawcett (2008) elogia el trabajo pionero de Castel. Este autor brinda una definición de las reglas del generador de la léxico-gramática de Cardiff en un lenguaje de marcas extensible (XML) con el objetivo de facilitar y ampliar el desarrollo de la gramática (Castel, 2007). Más recientemente, se ha presentado una micro-gramática sistémico-funcional para los clíticos en español (Castel, 2008). Existen, desde luego, otros estudios funcionales importantes, por ejemplo el análisis del Tema en español (MontemayorBorsinger, 1998) y variadas descripciones sobre el Tema y la modalidad en lenguas diferentes del inglés (LOTE) y de comportamiento similar al español, como el portugués, el italiano y el francés (Gouveia \& Barbara, 2005; Salvi \& Vanelli, 2004; Banks, 2003; Caffarel, 2004, 2006).

Los conceptos fundamentales de Cardiff se respaldan en la idea de que la cláusula consta de una variada trama de significados y es desde luego a Halliday a quien se le debe esa concepción del lenguaje (Fawcett, 1999). Halliday propuso la existencia de tres metafunciones (una perspectiva 'trinocular') y, a partir de ello, Fawcett (2008: 216) enunció explícitamente el "principio multifuncional del lenguaje”. En efecto, para Fawcett son más de tres las funciones que están presenten de forma simultánea en la cláusula. Así, el sistema de una lengua natural como el castellano permite expresar diversos significados al mismo tiempo en una única estructura.

La metodología del dialecto de Cardiff permitirá explicar 'cómo' se manifiestan los significados experiencial, interpersonal y textual en la cláusula española. Este dialecto reconoce otros cinco tipos principales de significados y tres significados secundarios, pero al menos por ahora nos restringiremos a los significados experiencial, interpersonal y textual, que son fundamentales para cualquier enfoque sistémico-funcional del lenguaje, más allá de las diferencias entre una escuela y otra (ver los trabajos más representativos de la Escuela de Sydney y de sus raíces, en particular los de Halliday, 1967a, 1967b, 1968, 1970, 1978, 1985, 1994; Halliday \& Matthiessen, 2004). 
En los incisos que siguen se mostrará que un diagrama arbóreo bidimensional capta la información necesaria de un complejo multidimensional de tipos de significado. Consideremos una oración-texto que tuvo lugar en un contexto social específico. El concepto de 'oración-texto' se refiere a un texto o fragmento de texto cuya estructura se corresponde con una unidad gramatical. Con esta noción (pertinente para nuestro análisis) queda en claro la vieja idea funcionalista según la cual la estructura y la función lingüística son dos dimensiones de un único y gran fenómeno.

En nuestra oración-texto una joven, Leticia, está hablando acerca de su bicicleta que tiene la cadena rota. Debido a esto, tendrá que ir al trabajo en autobús. Su mamá, Bruna, le dice que el problema de la bicicleta tiene solución porque otro hijo de ella y hermano mayor de Leticia, Juan, sabe arreglar bicicletas: “Ese problema tiene solución. Juan arregla bicicletas”. Considérese entonces la segunda 'oración-texto' de Bruna:

(1) Juan arregla bicicletas.

Según Fawcett (2008), para efectuar un análisis abarcador de cualquier oración-texto es conveniente recurrir a diagramas arbóreos (como los de las Figuras 1, 2 y 4, que aparecen más adelante en los apartados 1,2 y 5 , respectivamente). Por medio de estos diagramas se muestra que la estructura de la cláusula es como una fuerte soga de cáñamo, que consta de muchas hebras, cada una de las cuales a su vez está hecha de innumerables fibras. Dicho de otro modo, toda cláusula expresa varias hebras de significado, y cada una de ellas manifiesta una función distinta e incluye subsistemas más finos o delicados. Estas diferentes hebras de significado (y sus fibras más pequeñas) conforman un tejido muy compacto y, todas juntas, configuran lo que percibimos como una entidad unificada: la cláusula como un todo. En el contexto de este análisis, las categorías gramaticales-funcionales puestas en juego ('Sujeto', 'Operador', ‘Auxiliar', 'Sujeto-Tema', etc.) se irán definiendo junto al método de reconocimiento.

\section{Transitividad}

Una de las hebras de significado más importantes es la TRANSITIVIDAD, una red sistémica por medio de la cual se manifiesta la función experiencial del lenguaje en la estructura de la cláusula. No todas las comunidades usan el lenguaje para organizar salidas de pesca costera en lanchitas amarillas, como ocurre en el caso concreto de los pescadores artesanales del Puerto de Mar del Plata. Sin embargo, en todas las comunidades humanas el lenguaje se usa para hablar acerca del mundo, para organizar la experiencia. Así, en el caso concreto del castellano, la TRANSITIVIDAD define el rango de tipos de Procesos que es posible expresar y también los Participantes que se 
asocian a esos Procesos. Corresponde además a la TRANSITIVIDAD la caracterización de los diversos tipos de Circunstancias que, opcionalmente, pueden ofrecer precisiones sobre el evento que la cláusula realiza.

A lo largo de este trabajo, se emplearán letras en VERSALES para dar cuenta de sistemas semánticos, por ejemplo, Modo; en tanto que las realizaciones de opciones particulares dentro de cada sistema se escribirán con mayúscula y en fuente estándar, por ejemplo, Modo, entendido como 'dadora de información', 'buscadora de polaridad', etc., en referencia a una cláusula determinada.

El Proceso expresado es parte del significado experiencial, y todo Proceso es el pivote de una situación a la que se hace referencia. Puede decirse entonces que en la situación 'Juan arreglando bicicletas' el Proceso es 'arreglar'. En lo que respecta al nivel de la forma, el pivote de la unidad sintáctica que constituye la cláusula es el Verbo Principal 'arregla'. Es plausible sugerir que en castellano también se da el "principio más valioso" del análisis sintáctico del inglés (Fawcett 2008: 219): “Hay un solo verbo principal por cláusula”. En la Gramática de Cardiff, el Verbo Principal [M, del inglés Main Verb] es un elemento de la estructura de la cláusula y se relaciona directamente con muchos aspectos de los significados y las estructuras de la cláusula, por ejemplo con la expresión del Proceso y de los Roles Participantes [RPs]. Se ha dicho ya que la Gramática de Cardiff adopta un enfoque funcional para el análisis de cualquier lengua. En este contexto, es plausible la hipótesis de que las unidades de la sintaxis española se vinculen directamente a unidades de la semántica (si bien esto no entraña que dicha correspondencia deba ser uno-a-uno). Así como M expresa típicamente el Proceso, el Sujeto [S] y el Complemento [C] de la cláusula expresan típicamente los RPs.

A continuación, en la Figura 1 se ilustra la manifestación de la hebra experiencial del significado a partir de la estructura sintáctica elemental del Ejemplo (1).

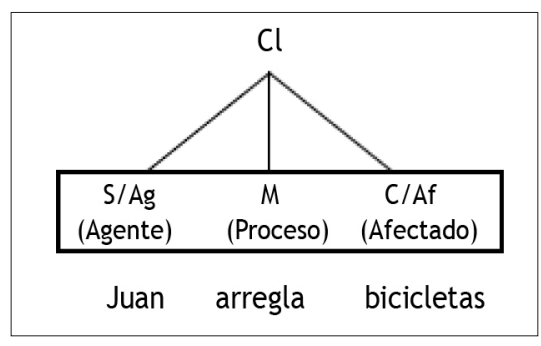

Figura 1. La tRANSITIVIDAD en el Ejemplo (1). 
El M 'arregla' expresa el Proceso. El Sujeto 'Juan' expresa uno de los dos RPs cuya aparición resulta esperable por el Proceso 'arreglar': 'alguien arregla algo'. Se sugiere que 'bicicletas' es el Complemento de esta estructura sintáctica porque también expresa 'el otro’ RP 'esperable' en términos del Proceso ‘arreglar' (otra vez: ‘algo es lo arreglado por alguien').

Las siguientes hipótesis (que establecen una relación directa entre unidades sintácticas y significados) parecen muy plausibles:

- El M español siempre expresa el Proceso.

- El S español siempre expresa un RP.

- El C español siempre expresa un RP, distinto del S.

El Complemento expresa un RP porque resulta necesario para 'complementar' el significado expresado por el Proceso. En el Ejemplo (1), 'arreglar' es una acción, mientras que el Sujeto está ‘amalgamado' con el rol Agente (el RP que 'efectúa' el Proceso) y el Complemento se amalgama con el Rol Afectado (i.e., el RP sobre el que recae la acción).

\section{Modo}

Otra función primordial del lenguaje es la de expresar significado interpersonal, lo que equivale a decir que en todas las culturas se emplea el lenguaje para establecer y mantener relaciones sociales mediante la asignación de roles comunicativos. También en español, el principal tipo de significado interpersonal se expresa en la cláusula por medio del sistema de Modo. Por ejemplo, en (1), Bruna se asigna a sí misma el rol comunicativo de 'dadora de información' y le asigna a su hija Leticia el rol de 'receptora de información'. En cambio, cuando Bruna le dice en (2) a su hijo Juan, los roles comunicativos son distintos.

(2) Arreglá la bicicleta de Leti, Juan.

En (2), Bruna se asigna a sí misma el rol de 'promotora de una acción' y a su hijo Juan el de 'receptor de una orden'. Los roles mencionados en estas líneas configuran vínculos interpersonales: Dar o recibir una orden, por ejemplo, son indicadores de roles comunicativos, interpersonales (y aun roles sociales). La cláusula castellana permite expresar ese significado, por ejemplo, mediante el indicativo o el imperativo (que son modos verbales y no el Modo mismo de la cláusula, aunque, como se ve, aportan lo suyo para expresarlo).

En el apartado 1 ha sido relativamente fácil establecer un paralelismo entre la TRANSITIVIDAD del castellano y la del inglés, y muy posiblemente lo mismo pueda hacerse con las otras lenguas del mundo. Es imposible, en cambio, trazar una analogía semejante entre el Modo del inglés y 
el español. En la Gramática de Cardiff, el Sujeto inglés se identifica a partir del ordenamiento de los elementos estructurales 'Sujeto' [S] y 'Operador' [0]. Ejemplos como (3a) y (3b) ilustran la cuestión de una forma característica. Puede definirse al Operador como la unidad lingüística que sirve para que la cláusula inglesa tenga flexión; el Operador (que muy frecuentemente es un auxiliar) es la unidad lingüística cuyo cambio de orden de declarativa a interrogativa permite reconocer el Modo. En los ejemplos de (3) el Operador está explayado por el auxiliar will.

(3a) They will come. [S O M] $\rightarrow$ S O significa ‘dadora de información' en inglés.

(3b) Will they come? [O S M] $\rightarrow$ O S significa 'buscadora de información' en inglés.

Para entender los breves análisis de $(3 a)$ y (3b) primero debe considerarse que en cualquier cláusula inglesa no puede haber más que un único Sujeto y un único Operador. Antes de identificar el Sujeto, debe identificarse el Operador (si es que existe alguno). En este punto también conviene destacar que en la Gramática de Cardiff se usa 'explayar' (expound) como un 'concepto técnico' que caracteriza el vínculo entre un elemento de la estructura arbórea y el ítem o los ítems que aparecen debajo en el diagrama (Fawcett, 2008). Así, al caracterizar 'el significado del Sujeto en la Gramática’, Fawcett (1999) señala que, por ejemplo, el Sujeto de la cláusula inglesa Shall we eat it? ['¿Podemos comérnoslo?'] está 'explayado’ (expounded) por we, mientras que el Operador está 'explayado’ por shall.

Siempre para el caso del inglés, Fawcett (1999) afirma que el reconocimiento del Operador no es problemático, puesto que las palabras que lo explayan se agrupan en un pequeño conjunto muy fácil de memorizar. En efecto, el Operador inglés es uno de estos verbos modales: can, must, may, might, shall, should, will, would. En este contexto, el verbo be resulta único porque $M$ se amalgama con 0 , formando así la unidad $0 / M$. Por último, cuando no existe ningún Operador explayado en la cláusula, el auxiliar do es el Operador por defecto y sirve para transformar una 'dadora de información' en ‘buscadora de polaridad'.

A partir de consideraciones como las del párrafo anterior, Fawcett concluye que la disposición sintáctica del Sujeto y el Operador es determinante para la expresión del Modo en inglés, como se muestra en los ejemplos (3a) y (3b). No ocurre lo mismo en castellano: Las cláusulas del conjunto integrado por (1) y (4a)-(4e) son todas dadoras de información si se las enuncia con un acento nuclear descendente, que (a grandes rasgos) es similar al "tono 1" del inglés (Halliday \& Matthiessen, 2004: 141). La posición del Sujeto (que está subrayado en los ejemplos) no expresa el Modo de estas cláusulas. El acento nuclear se indica mediante negritas:

(1) Juan arregla bicicletas.

(4a) Juan bicicletas arregla. 
(4b) Bicicletas arregla Juan.

(4c) Bicicletas Juan arregla.

(4d) Arregla bicicletas Juan.

(4e) Arregla Juan bicicletas.

Sin embargo, todas ellas bien podrían ser 'buscadoras de información' si se las enunciara con un acento nuclear ascendente, más o menos similar al "tono 2" del inglés (Halliday \& Matthiessen, 2004: 141). El conjunto (5a)-(5f) representa ortográficamente esta alternativa. (El Sujeto vuelve a estar subrayado, y los acentos nucleares figuran nuevamente en negritas).

(5a) ¿Juan arregla bicicletas?

(5b) ¿Juan bicicletas arregla?

(5c) ¿Bicicletas arregla Juan?

(5d) ¿Bicicletas Juan arregla?

(5e) ¿Arregla bicicletas Juan?

(5f) ¿Arregla Juan bicicletas?

En la escritura, varios de los ejemplos anteriores, en especial (4a), (4c), (4e), (5b-d), pueden parecer de dudosa aceptabilidad. Sin embargo, todas las oraciones-texto de (4) y (5) son perfectamente aceptables en situaciones de diálogo donde se las enuncie con la entonación apropiada. Se incluyen estos casos con una importante variación en el orden de palabras por dos razones fundamentales: (i) como se ha dicho, ayudan a mostrar que la posición del Sujeto no expresa el Modo de una cláusula castellana; y (ii) abren la posibilidad de relacionar los significados temáticos con los significados informativos ('lo dado' y 'lo nuevo'), un aspecto que se tratará (aunque de manera muy general) en el apartado 5 y a partir de uno de los últimos trabajos de Fawcett (en prensa).

El Sujeto castellano parece expresar al menos dos tipos de significado interpersonal, o fuertemente asociados al interpersonal: (a) un significado 'gramaticalmente' redundante, como réplica de la conjugación de la forma amalgamada $\mathrm{M} / \mathrm{O}$; (b) un conjunto de significados 'comunicativamente' relevantes. Lo que se quiere decir por medio de (a) es que el Sujeto español no es necesario para expresar el Modo ni la conjugación; la flexión verbal es suficiente para ello. Sin embargo, cuando S está explayado, sí contribuye a (re)expresar el significado que ya está en la amalgama $M / O$. Cuando existe un verbo auxiliar, que se representa por medio de X, el S está explayado en la amalgama X/O. En el conjunto de (b) podrían incluirse significados de cortesía o significados afectivos. En la Gramática de Cardiff, se reconoce la existencia de una hebra de significado AfEctivo independiente. Tal vez parte de los significados 'comunicativamente rele- 
vantes' que aquí apuntamos se correspondan con esta hebra. Así, la explicitud del Sujeto puede indicar, por ejemplo, un fuerte compromiso del hablante con respecto a lo que está diciendo, como en el Ejemplo (6).

(6) Yo te quiero.

Este significado interpersonal/afectivo puede parangonarse al que a veces se sugiere por medio de la presencia del Vocativo, como en el Ejemplo (7).

(7) Te quiero, Juana.

En efecto, resulta plausible la hipótesis de que el Sujeto español exprese, como el Vocativo, 'algún' significado interpersonal (o tal vez fuertemente ligado al interpersonal). Dicho toscamente, la explicitud del Sujeto o del Vocativo puede agregar matices de significado interpersonal o afectivo a la cláusula. De todos modos, sigue siendo fundamental que la disposición sintáctica del Sujeto respecto del Operador no es de manera alguna lo que expresa el Modo (adviértase aquí otra similitud del Sujeto con el Vocativo, puede aparecer en cualquier lugar de la cláusula sin alterar el Modo).

Al parecer el Sujeto en castellano se inclina marcadamente a la expresión del significado textual. Pueden mencionarse tres ejemplos que se desarrollan más ampliamente en el inciso 3: (i) con frecuencia el Sujeto español es un recurso cohesivo muy importante (porque crea o cambia referencia); (ii) resulta necesario a la hora de evitar ambigüedades en ciertas cláusulas potencialmente ambiguas, como 'Caminaba por el parque' (donde el verbo podría corresponder a la primera o a la tercera persona del singular); (iii) también es necesario o útil para ampliar el desarrollo temático por medio del vínculo entre el rema de una cláusula y el tema de la siguiente.

Ahora bien, ¿qué es lo que determina los roles comunicativos en la cláusula española? Una hipótesis plausible es la que se representa en la Figura 2. El Modo castellano se expresa tanto por medio del verbo conjugado como por medio de la entonación. La morfología (a través de los rasgos flexivos) y la fonología suprasegmental (a través de la entonación) desempeñan juntos un papel decisivo en la expresión de 'dadoras de información', 'buscadoras de información' 'promotoras de acción', etc. Los rasgos flexivos de M siempre están adheridos al Operador, lo que da como consecuencia la amalgama M/O. Los ítems léxico-gramaticales que expresan el Modo están en negrita: 


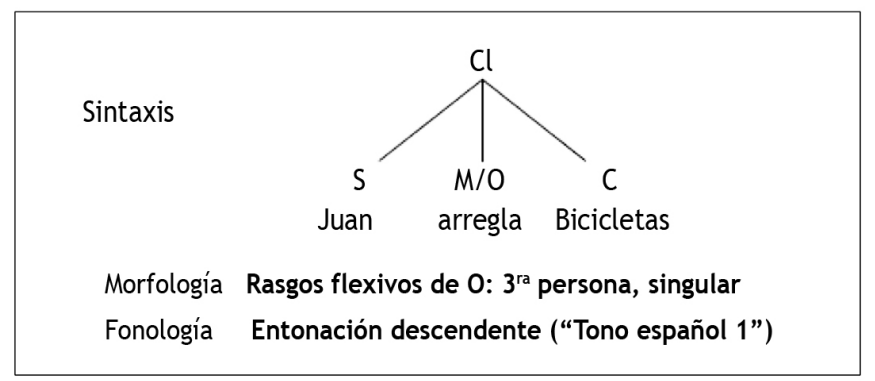

Figura 2. El Modo en el Ejemplo (1).

Podría añadirse que en castellano no existen Operadores independientes como los que establece la Gramática de Cardiff para el caso del inglés. El Operador castellano puede definirse como la unidad lingüística que expresa la flexión de la cláusula y se amalgama con el Verbo Principal (así lo muestra la Figura 2) o con el Auxiliar X (ver Figura 4). Véanse también al respecto los ejemplos (8) y (9). En español no existen Operadores independientes ni Operador por defecto, porque el Operador tampoco participa en la determinación del Modo.

(8) Perón ha muerto.

$\mathrm{S}=$ Perón; $\mathrm{X} / \mathrm{O}=$ ha; $M$ = muerto.

(9) Juan puede arreglar bicicletas.

$\mathrm{S}=$ Juan; $\mathrm{X} / \mathrm{O}=$ puede; $\mathrm{M}=$ arreglar; $\mathrm{C}=$ bicicletas.

El análisis aquí desplegado no permite aplicar los Tests del Sujeto que se han propuesto para el inglés. Por ejemplo, Halliday y Matthiessen (2004) recurren a las preguntas con coletilla (tag questions) y Fawcett $(1999,2008)$ tiene en cuenta las posiciones del Sujeto y el Operador, tal como se ha explicado en este inciso. Para Fawcett (2008:231), el Sujeto inglés “es la palabra o conjunto de palabras que, por aparecer antes del Operador, muestra si la cláusula es una dadora de información o una buscadora de información". La definición, como podrá advertirse, es claramente funcional.

En síntesis, los lineamientos de la Gramática de Cardiff nos permiten emprender una caracterización del Modo castellano y proponer la necesidad de un Test del Sujeto para nuestra lengua. De eso se trata el apartado que sigue. 


\section{Un Test del Sujeto para el español}

No se ha explicado aún ‘cómo’ reconocer que ‘Juan’ es el Sujeto en el Ejemplo (1). Con el objetivo de dar esa explicación tomaremos prestado algún concepto de la gramática estructural (Rossetti \& Zamudio, 1984; Barrenechea \& Rossetti, 1986; Kovacci, 1992). El mismo Fawcett (1999) hace explícito que él decide recurrir a nociones tradicionales con el fin de elaborar un Test del Sujeto para inglés. De todas maneras, la caracterización del Sujeto sigue siendo sistémica y funcionalista. Ya en la Figura 2 se pone de manifiesto que una caracterización sistémicofuncional del Sujeto debe considerar los rasgos flexivos que determinan la concordancia, por ejemplo la concordancia morfológica entre la flexión del verbo y el sustantivo-sujeto (i.e., el sustantivo que es parte del grupo que a su vez 'llena' el Sujeto). La unidad verbal conjugada es la amalgama M/O o la amalgama X/O. Estamos ahora en condiciones de presentar los dos primeros pasos de 'Un Test del Sujeto para el español':

Paso 1: Encuentre la forma conjugada:

1.1. Encuentre la amalgama $X / 0$, si no, pase a 1.2.:

1.2. Encuentre la amalgama $M / O$.

Paso 2: Encuentre el sustantivo que concuerda con la forma conjugada (1.1. o 1.2.).

Los ejemplos (10)-(13) ejemplifican algunas relaciones de concordancia basadas en la coincidencia de persona y número entre el Sujeto y la flexión, i.e., entre el Sujeto y la amalgama $\mathrm{X} / \mathrm{O}$ o la amalgama $\mathrm{M} / \mathrm{O}$.

\begin{tabular}{|c|c|}
\hline $\begin{array}{l}(10) \\
\frac{\text { Juan }}{L}[S] \frac{\text { arregla }}{J}[M / 0] \text { bicicletas } \\
\text { Conc.: } 3^{\text {ra }} \text { persona, singular }\end{array}$ & $\begin{array}{l}(12) \\
\frac{\text { Juan }}{L}[S] \text { puede }[X / O] \text { arreglar bicicletas } \\
\text { Conc.: } 3^{\text {ra }} \text { persona, singular }\end{array}$ \\
\hline $\begin{array}{l}\text { (11) } \\
\text { A Juan le gustan [M/O] las bicicletas [S] }\end{array}$ & $\begin{array}{l}\text { (13) } \\
\text { A Juan siempre le han [O/Ml oustado las bicicletas [S] }\end{array}$ \\
\hline $\begin{array}{l}\text { A Juan le gustan }[M / 0] \text { las bicicletas }[S] \\
\text { Conc.: } 3^{\text {ra }} \text { persona, plural }\end{array}$ & $\begin{array}{c}\text { A Juan siempre le han }[\mathrm{O} / \mathrm{M}] \text { gustado las bicicletas }[\mathrm{S}] \\
\text { Conc.: } 3^{\text {ra }} \text { persona, plural }\end{array}$ \\
\hline
\end{tabular}

En este análisis no puede dejarse de lado la muy frecuente opción del 'Sujeto Tácito'. El Sujeto no se explaya pero es recuperable en términos de la flexión. Los ejemplos (14) y (15) pueden resultar útiles para mostrar que el Sujeto español se comporta un modo diferente del Sujeto inglés; este último casi siempre se realiza de forma manifiesta, seguramente por su importancia para la expresión del significado interpersonal. Por su parte, no es raro que el Sujeto castellano 
esté ausente, al menos en el nivel de la forma. Por ello se sugiere que el Sujeto español es 'gramaticalmente redundante'. La información gramatical de la flexión verbal permite omitir el Sujeto; y esa misma información también permite reconocerlo.

Estudiamos [M/O] Lingüística (Sujeto Tácito: “Nosotros”)

Conc.: $1^{\text {ra }}$ persona, plural

Habíamos $[X / O]$ estudiado Lingüística (Sujeto Tácito: “Nosotros”)

Conc.: $1^{\text {ra }}$ persona, plural

En consecuencia, el segundo paso de este Test del Sujeto puede no ser posible: De ser así, el analista de la gramática castellana debe ir a un tercer paso:

Paso 3: (Si no es posible el Paso 2) haga explícito un pronombre personal que concuerde con la unidad hallada en el Paso 1: Es lo que se hizo en los ejemplos (14) y (15).

Estos tres pasos no solo son suficientes para explicar e identificar el Sujeto en un porcentaje altísimo de cláusulas castellanas, sino también para explicar los casos (potencialmente) ambiguos como (16):

(16) Maneja Mercedes.

Interpretación 1 del ejemplo (16):

Paso 1: Encuentre la forma conjugada: Maneja (M/O)

Paso 2: Encuentre el sustantivo que concuerde con la forma conjugada: Mercedes

(17) Maneja [M/O] Mercedes [S].

Conc.: $3^{\text {ra }}$ persona, singular

Sin embargo, para la secuencia (16), también puede proveerse la Interpretación 2: 
Interpretación 2 del ejemplo (16):

Paso 1: Encuentre la forma conjugada: Maneja (M/O)

Paso 2: Encuentre el sustantivo que concuerde con la forma conjugada: No es posible.

Paso 3: Haga explícito un pronombre que concuerde con la unidad hallada en el Paso 1: él/ella.

(18) Maneja [M/O] Mercedes (Sujeto Tácito: ‘Él/Ella’)

Conc.: $3^{\text {ra }}$ persona, singular

El Test del Sujeto en español permite tratar casos potencialmente ambiguos como (16). Por supuesto, las oraciones se realizan en textos orales o escritos y los textos se producen en contextos sociales específicos. Por ello, un hablante dice (16) pero quiere decir o bien (17) o bien (18), y no los dos a la vez (con excepción de ciertos usos humorísticos, o de otra índole, que adrede explotan la ambigüedad referencial). En situaciones reales, el oyente probablemente entienda lo que el hablante quiso decir; los textos breves de (19) y (20) muestran que una oración como (16) no resulta ambigua en un contexto puntual.

(19) Maneja Mercedes. Durmió bien y tiene más experiencia que yo en la ruta.

(20) Maneja Mercedes. También Porsche, Volvo y Ferrari. Es una mujer muy rica.

La cláusula (16) no será ambigua en un contexto específico como los de (19) y (20). Un hablante nativo de español será capaz de usar el Sujeto Tácito o el Sujeto Explícito de acuerdo con los significados que necesite expresar y parece razonable sugerir que los significados asociados a la forma Sujeto Explícito en español son eminentemente textuales, más que interpersonales.

Por último, se sabe que un grupo relativamente pequeño pero importante de oraciones castellanas son impersonales porque 'no tienen Sujeto'. Esto es, oraciones como las de los ejemplos (21)-(24) no permiten ni Sujeto Explícito ni Sujeto Tácito, definitivamente carecen de Sujeto.

(21) Hay problemas.

(22) Hace frío.

(23) Llueve.

(24) Se castigó a los culpables.

Al parecer, el Test del Sujeto en español también es útil para caracterizar la expresión de impersonalidad cuando se elige la 'falta' de Sujeto (que por supuesto no debe confundirse con la simple ausencia indicada a través del 'Sujeto Tácito'). En estos casos, ni el segundo ni el 
tercer paso del Test del Sujeto en español son posibles. Por lo tanto, el analista de la gramática castellana deberá recurrir a un cuarto paso.

Paso 4: Debe concluirse que la cláusula es impersonal (no tiene Sujeto).

Consideremos los ejemplos (21)-(24) en el análisis de (25).

(25) Test del Sujeto para las cláusulas impersonales (21)-(24):

Paso 1: Encuentre la forma conjugada: 'hay’ (21), ‘hace’ (22), ‘llueve’ (23), ‘castigó' (24).

Paso 2: Encuentre el sustantivo que concuerda con la forma conjugada: No es posible.

Paso 3: Haga explícito un pronombre que concuerde con la unidad hallada en el Paso 1: No es posible.

Paso 4: Debe concluirse que la cláusula es impersonal (no tiene Sujeto).

La sola existencia de cláusulas impersonales en español constituye otra razón a favor de la hipótesis sugerida en el ítem 2. El Sujeto español (ya sea porque es Tácito o Explícito o por su disposición sintáctica respecto de 0 o M) no expresa el Modo. Un ejemplo visible de esto es que las no poco frecuentes oraciones del conjunto (21)-(24) no tienen Sujeto, pero sí expresan Modo. Buena parte del análisis desplegado en este inciso puede resumirse por medio de la Figura 3.

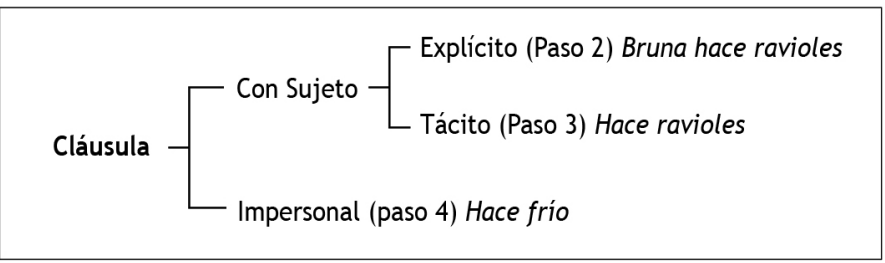

Figura 3. Un sistema de reglas de realización muy simple para el Sujeto español (vinculado al Test del Sujeto).

Conviene aclarar que en la Gramática de Cardiff, la forma lingüística (sintaxis, morfología, léxico y puntuación o entonación), no se conceptualiza como una red sistémica, lo que marca una diferencia sustantiva con la Gramática de Sydney. En la Figura 3 se exponen reglas de realización que forman parte del potencial de la forma. En términos de Fawcett (2008), las redes del sistema con rasgos semánticos constituyen el potencial de significado de una gramática 
sistémico-funcional y por ello permiten la selección de la expresión de rasgos semánticos (una instanciación en el nivel de significado). Por su parte, las reglas de realización como las de la Figura 3 integran el potencial de la forma de una gramática sistémico-funcional y dan lugar a 'estructuras arbóreas propiciamente rotuladas' (richly labeled tree structures), que no son otra cosa que instanciaciones en el nivel de la forma, como, por ejemplo, la oración-texto: 'Juan arregla bicicletas', analizada en las Figuras 1 y 2.

\section{Tema}

Se entiende aquí que el Tema es el sistema por medio del cual se expresa aquello de lo que trata la cláusula. Por su parte, el Tema de una cláusula es la forma lingüística a través de la que se expresa aquello de lo que trata la cláusula. Las definiciones nos sitúan en el punto de vista textual, es decir, en la concepción de la cláusula como mensaje o como fragmento de un texto. Por ejemplo, el significado transitivo de ‘Aldosivi jugó con Alvarado' y 'Alvarado jugó con Aldosivi' es el mismo, pero en la primera cláusula-mensaje el Tema es 'Aldosivi', mientras que en la segunda el Tema es 'Alvarado'.

En este contexto, la noción de 'Sujeto-Tema' (Fawcett, 1999) también parece muy pertinente para el caso del castellano. El Sujeto-Tema es un sub-tipo particular de tema en las cláusulas de muchas lenguas (como el inglés y el castellano), es aquello de lo que trata la cláusula en tanto elemento gramatical que concuerda con la flexión del verbo. En efecto, el elemento que ocupa la posición de Sujeto es importante desde el punto de vista temático. En el caso del castellano, explicitar o no el Sujeto son dos opciones, dentro del potencial de la forma, cuya condición de entrada en la red semántica es el Sujeto. El Sujeto-Tema se superpone con el Tema cuando está en posición inicial, pero cuando el Sujeto-Tema no ocupa la posición inicial existe otro elemento que también se considera Tema.

En el apartado correspondiente al Modo se sugirió que el Sujeto castellano se ha desarrollado más para la expresión de significados textuales que para la expresión de significados interpersonales; Fawcett (1999) destaca que en la Gramática de Cardiff el potencial de la forma (las reglas de realización) está conectado con el potencial de significado (la red del sistema con rasgos semánticos). Existen buenas razones entonces para interpretar que cualquier Rol Participante amalgamado con el Sujeto también será Sujeto-Tema, de manera que el concepto ya está representado en las estructuras arbóreas propiciamente rotuladas de las Figuras 1 y 2.

Tal como lo muestra la Figura 3, 'Sujeto Explícito' es una opción en el nivel de la forma. Esto es, explicitar el Sujeto en castellano consiste en darle el status de un tipo especial de Tema. 
Si, además, el Sujeto-Tema aparece al principio de la cláusula, también estará 'tematizado', i.e., será el Tema propiamente dicho. A propósito, el Tema propiamente dicho de cada cláusula también puede comprender otros elementos formales: interpersonales, como el vocativo, o textuales, como las conjunciones. En los ejemplos de (26a)-(26c) y de (27a)-(27b), el SujetoTema está subrayado y el elemento tematizado se escribe con bastardilla.

(26a) Bruna hace ravioles.

(26b) Ravioles hace Bruna.

(26c) Hace ravioles Bruna.

(27a) Hace ravioles [Sujeto 'Tácito']

(27b) Ravioles hace [Sujeto 'Tácito']

En los dos ejemplos de (27) el Sujeto está 'Tácito'. En términos de la Gramática de Cardiff, digamos que no se lo ha explayado pero que es recuperable (en español, de hecho, el total de su información gramatical estará de todas formas presente en la flexión verbal). Debido justamente a su ausencia, el Sujeto Tácito no está tematizado. Por su parte, el Sujeto Explícito es un Sujeto-Tema porque el hablante considera que existen buenas razones comunicativas como para explayarlo, por ejemplo, la necesidad afectiva de destacar su presencia o la de mostrar un cambio de referencia. Un Sujeto-Tema que además está tematizado, como en (26a), puede concebirse como aquello de lo que trata la cláusula. Sin embargo, un Sujeto-Tema no tematizado como en (26b) también es un elemento de peso temático porque el hablante considera que existen buenas razones para explicitar el Sujeto.

A partir de este análisis, la Gramática de Cardiff nos ayuda a entender que podría haber otros casos de tematización que acarrearían significados diferentes y tal vez mucho más fuertemente marcados, por ejemplo, la tematización de un Complemento, tal como ocurre en los ejemplos (28) y (29), donde el Sujeto-Tema está otra vez subrayado y el Tema en bastardilla.

(28) Los ravioles los hace Bruna.

(29) Los ravioles yo los adoro.

La tematización del Complemento es una opción que, dado un contexto apropiado, resulta comunicativamente pertinente. Desde luego, existen otros tipos de Tema como la tematización de Circunstanciales u otros significados realizados por medio de Adjuntos. Fawcett (1999) señala que, debido a la inmensa variedad de propósitos discursivos por los cuales se tematizan los elementos, es razonable no sugerir ninguna conclusión acerca los significados del Tema más 
allá de que es 'el pilar sobre el que se apoya el mensaje' (Halliday, 1968, 1970). De todas maneras, sí puede sugerirse que el Sujeto-Tema es un tipo muy importante de Tema en castellano. Como se ha dicho, el Sujeto español puede tener algún significado interpersonal pero ninguno que contribuya a la determinación del Modo. Al parecer, el Sujeto castellano se inclina más a la expresión del significado textual. En el inciso 2 se mencionaron tres casos que ahora se ejemplificarán.

En primer lugar, el Sujeto español es un recurso cohesivo muy importante en la medida que crea o cambia referencia, como en el texto del Ejemplo (30).

(30) Javier Gómez se llevó la pelota por la izquierda para la tribuna donde estaba la hinchada de Racing. No había más que un defensor en ese costado, $\left[\emptyset_{1}\right]$ lo eludió, $\left[\varnothing_{1}\right]$ llegó hasta el fondo y $\left[\varnothing_{1}\right]$ sacó centro rasante. Por el medio del área venía de frente Corti. $\left[\varnothing_{2}\right]$ Conectó el pase y $\left[\varnothing_{2}\right]$ mandó la pelota al fondo del arco. El Vasco Solaberrieta estaba cerca y $\left[\varnothing_{3}\right]$ fue corriendo a abrazarlo. [Miguel di Bartolomeo, comentario del partido Racing de Córdoba-Aldosivi de Mar del Plata, Radio del Puerto, 9 de julio de 2005].

En el Ejemplo (30), que se ha ajustado algo artificialmente a las pautas de la ortografía, la aparición de un nuevo Sujeto se marca con negritas. La primera mención de 'Javier Gómez' se hace por medio de Sujeto Explícito (de lo contrario, es obvio que no sabríamos de quién se está hablando). Luego existen tres casos de elipsis $\left[\emptyset_{1}\right]$ (y Sujeto Tácito) hasta que se cambia la referencia y el Sujeto pasa a ser otro: 'Corti'. Siguen dos casos de elipsis (y Sujeto Tácito) hasta que vuelve a cambiarse de Sujeto con la mención de otro jugador: 'El Vasco Solaberrieta'.

El Sujeto Explícito también resulta necesario para evitar ambigüedades. Las cláusulas de (31a) y (31b) evitan la ambigüedad en la que se podría caer al emitir el Ejemplo (31).

(31) Trabajaba en la municipalidad.

(31a) Yo trabajaba en la municipalidad.

(31b) Mi viejo trabajaba en la municipalidad.

Como un tercer ejemplo (puede haber muchos más) considérese el desarrollo temático por medio del vínculo entre el rema de una cláusula y el tema de la siguiente, tal como se da en el ejemplo (32). Se trata de una instancia de lo que Danes y otros lingüistas checos denominaron 'progresión temática lineal simple', algo así como una gradual tematización de remas (Danes, 1974). El texto de (32) es un fragmento de un folleto con la explicación de la estructura de un equipo del Pueyrredón Rugby Club de Mar del Plata (Gil, 2001: 265). Se subraya el tema de cada cláusula y el rema se escribe [entre corchetes].

(32) La tercera línea de forwards [está integrada por dos alas o wing-forwards y el octavo]. Los alas y el octavo [tienen numerosas responsabilidades]. Las más importantes 
[son la defensa y el apoyo]. Defensa y apoyo y apoyo [son en rugby tareas muy delicadas porque hacen a la estructura general de un equipo]. Y un equipo [es un reflejo de la sociedad]...

Obsérvese que en el rema de una cláusula se trata como el tema de la siguiente. El Sujeto Explícito desempeña aquí un rol decisivo en cada uno de los mensajes expresados por la cláusula $\mathrm{y}$, desde luego, en el texto completo.

\section{Variaciones españolas}

A lo dicho en el inciso anterior debe agregársele lo siguiente: Fawcett (en prensa) señala que el concepto unificado de 'tema' en inglés puede definirse, y solo de manera aproximada, como el conjunto de varios tipos de significado 'temático' que comparten la característica de que se realizan en posiciones iniciales en la cláusula. Esto, sin embargo, no implica que todo elemento inicial sea el resultado de una tematización; los pronombres interrogativos y relativos, por ejemplo, no están en posición inicial porque se los haya tematizado. Están allí porque ese es el lugar donde se realizan. Después de analizar los muchos tipos de 'Tema' en inglés en términos sintácticos, semánticos y discursivos, Fawcett (en prensa) concluye que, en lo que hace al significado, cualquier generalización deberá orientarse a contemplar, por lo menos, los siguientes significados (en los ejemplos, el tema se subraya):

- 'Esto es de lo que estoy hablando', para el Sujeto-Tema, como en This gazebo was built by Sir Christopher Wren.

- 'Tengo fuertes sentimientos con respecto a esto', para un tipo de Rol Participante Marcado, como en Clever she may be, but a genius she is not.

- 'Aquí es donde debo corregir tu posible error', para lo nuevo contrastivo (contrastive newness), como en I've seen King Lear several times - (but) Coriolanus I haven't seen.

- 'Esta es la evaluación afectiva del hecho al que se hace referencia en la cláusula', para Adjuntos Afectivos como en Unfortunately she was late.

- ' 'Esta es mi evaluación de la probabilidad del evento', para Adjuntos de Validez como $\underline{\text { Per- }}$ haps he will come soon.

- Esta es mi evaluación de lo que puede esperarse como respuesta emotiva evocada por el evento', para los varios tipos de Tema Ampliado Evaluativo, como en It is difficult that she was late. 
- 'Esta es una circunstancia del evento en la cláusula que yo quiero que veas como especialmente importante', para Adjuntos experienciales como en Carefully, he opened the door o With his teeth he cut the rope.

- 'Estoy ubicando el tiempo o el lugar del hecho representado en la cláusula para las cláusulas principales subsiguientes hasta que sea necesario redefinir el tiempo y el lugar', In the garden at midnight they kissed each other.

- 'Estoy situando la escena para el próximo pasaje textual por medio de una especificación general del tiempo y el lugar de los hechos': Adjuntos de Posición del Establecimiento de la Escena de Tiempo y Lugar como In London last year I first met lke.

- 'Esta es la relación lógica entre la proposición siguiente y otra', para casos como Because he knew the answer, he was calm.

- 'Este objeto o persona es tanto nuevo como importante para este texto', para el Tema Ampliado Existencial, como en There was a tall man over by the bar.

Como se adelantó al final del inciso 2, el exhaustivo análisis de Fawcett (2008) para el inglés sirve de punto de partida para tratar ejemplos castellanos en los que existe una importante variación en el orden de palabras. Los ejemplos (33a)-(33e) pueden considerarse variaciones relativamente marcadas del ejemplo (1), que es no marcado.

(1) Juan arregla bicicletas.

(33a) Juan bicicletas arregla.

(33b) Bicicletas arregla Juan.

(33c) Bicicletas Juan arregla.

(33d) Arregla bicicletas Juan.

(33e) Arregla Juan bicicletas.

Independientemente de las variaciones sintácticas, ‘Juan' es el Sujeto, 'arregla' el Verbo Principal y 'bicicletas' el Complemento. En lo que respecta al significado experiencial, la TRANSITIVIDAD es la misma que la que se consigna en la Figura 1: el Proceso es ‘arreglar', ‘Juan' es el Agente y 'bicicletas' el Afectado. En términos del significado interpersonal, el Modo de todas estas cláusulas, con un acento nuclear descendente, es de ‘dadoras de información'. (Si se hubieran emitido con un acento nuclear ascendente habrían sido 'buscadoras de información'). Existen, por último, cambios pertinentes en lo referido al TEMA, puesto que aquello de lo que trata cada una de las cláusulas (33a)-(33e) varía según el elemento tematizado, i.e., en posición inicial. Lo importante aquí es que tres hebras fundamentales del significado (que tienen que ver con 
las metafunciones experiencial, interpersonal y textual) están presentes en la estructura de la cláusula castellana. Consideremos ahora el Ejemplo (34), donde se explaya un Verbo Auxiliar X: 'puede'.

(34) Juan puede arreglar bicicletas.

En la Figura 4 se muestran, por medio de una estructura arbórea propiciamente rotulada, la Transitividad, el Modo y el Tema del Ejemplo (34).

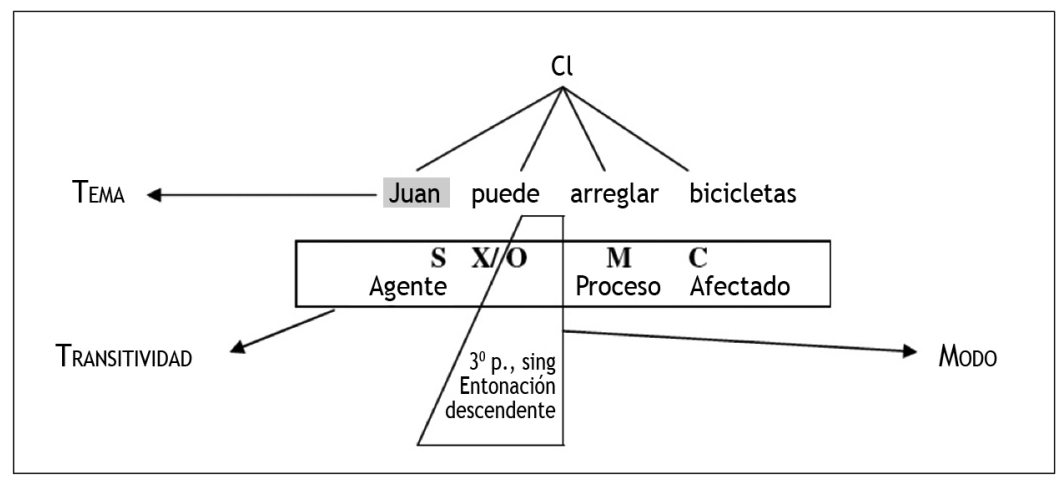

Figura 4. Transitividad, Tema y Modo en el Ejemplo (34).

Tal como se muestra a partir de (33a)-(33e) existen seis combinaciones posibles para los elementos del Ejemplo (1). Puesto que en el Ejemplo (34) existen cuatro elementos, las combinaciones posibles son los 24 casos de la Tabla 1. 
Tabla 1. Veinticuatro combinaciones sintácticas posibles de los elementos del Ejemplo (34).

\begin{tabular}{|l|l|}
\hline (1) Juan puede arreglar bicicletas & (13) Puede Juan arreglar bicicletas. \\
(2) Juan puede bicicletas arreglar. & (14) Puede Juan bicicletas arreglar. \\
(3) Juan arreglar bicicletas puede. & (15) Puede arreglar Juan bicicletas. \\
(4) Juan arreglar puede bicicletas. & (16) Puede arreglar bicicletas Juan. \\
(5) Juan bicicletas puede arreglar. & (17) Puede bicicletas Juan arreglar. \\
(6) Juan bicicletas arreglar puede. & (18) Puede bicicletas arreglar Juan. \\
\hline (7) Arreglar bicicletas Juan puede. & (19) Bicicletas puede arreglar Juan. \\
(8) Arreglar bicicletas puede Juan. & (20) Bicicletas puede Juan arreglar. \\
(9) Arreglar Juan bicicletas puede. & (21) Bicicletas arreglar puede Juan. \\
(10) Arreglar Juan puede bicicletas. & (22) Bicicletas arreglar Juan puede. \\
(11) Arreglar puede Juan bicicletas. & (23) Bicicletas Juan arreglar puede. \\
(12) Arreglar puede bicicletas Juan. & (24) Bicicletas Juan puede arreglar. \\
\hline
\end{tabular}

Se ha señalado que el trabajo de Fawcett (en prensa) podría ser un buen punto de partida para pensar la relación entre los significados informativos ('lo dado' y 'lo nuevo') y los significados temáticos. Precisamente, Fawcett entiende, al igual que Halliday, que en la cláusula inglesa el significado temático se realiza por medio de la secuencia de constituyentes y que el significado informativo se realiza por medio de la entonación (Fawcett, en prensa). Pero en la Gramática de Cardiff, donde se reconocen más hebras de significado, el 'Tema' es función del significado temático y lo ‘Dado' es función del significado informativo (tradicionalmente, Halliday consideró que las estructuras de Tema-Rema y Dado-Nuevo pertenecen a la metafunción y al significado textual de la cláusula).

Escapa desde luego a las posibilidades de este artículo la presentación exhaustiva de los diversos tipos de significados temáticos e informativos del español. Se exponen los ejemplos de la Tabla 1 para cumplir con tres objetivos muy simples: (i) reconocer que en efecto existe una importante posibilidad de combinaciones en el orden de palabras; (ii) apoyar la hipótesis de que el Sujeto no expresa el Modo de la cláusula española; (iii) advertir sobre la necesidad de explorar los seguramente muy diversos tipos de tema en español, de un modo análogo al que lo hace Fawcett para el inglés.

En efecto, resulta plausible la hipótesis de que la Gramática de Cardiff permitirá entender la compleja interacción entre los significados temáticos e informativos en el español. Tomemos, por ejemplo, dos casos de la Tabla 1. En el Ejemplo (1) 'Juan puede arreglar bicicletas', el 
Sujeto 'Juan' expresa tanto el Tema como lo Dado. En el Ejemplo (24) 'Bicicletas Juan puede arreglar’, el Tema es ‘bicicletas’ y ‘Juan’ es lo Dado (además del Sujeto-Tema). En este caso, 'bicicletas' puede muy bien expresar un significado de "lo nuevo contrastivo”, donde el hablante está comunicando que son precisamente bicicletas la clase de cosas que Juan puede arreglar, y no otras sugeridas antes por algún interlocutor.

Para la Transitividad y el Modo, en el total de ejemplos de la Tabla 1 aparecen los mismos elementos y las mismas funciones que se caracterizan en la Figura 4. También en todos estos casos 'Juan' es el Sujeto-Tema (aunque no en todos, claro está, aparece tematizado). Sería necesario investigar si algunos de estos 24 casos, en especial aquellos que están subrayados, son combinaciones aceptables. Tal vez las 24 combinaciones (aun las subrayadas) son aceptables y, en especial, funcionalmente pertinentes si se las emite con la entonación apropiada.

De todas formas, la conclusión es esta: la léxico-gramática y la entonación del castellano dan lugar a que los hablantes combinen 'los mismos elementos' en una cláusula para producir mensajes muy variados.

\section{CONCLUSIONES}

En primer lugar, los conceptos y la metodología de la Gramática de Cardiff constituyen un buen punto de apoyo para mostrar que la cláusula castellana es una estructura sintáctica que expresa varios significados al mismo tiempo. La representación de una estructura arbórea propiciamente rotulada (como en las figuras 1, 2 y 4) permite que el lingüista y aun una computadora (Castel, 2007) capten los rasgos semánticos que se han elegido. Esto es, una cláusula se genera a partir del potencial de significado del sistema lingüístico. En este sentido, los conceptos fundamentales de Sujeto S, Auxiliar X, Verbo Principal M y Complemento C no solo resultan útiles sino también necesarios para caracterizar las diversas hebras de significado en la cláusula castellana. Precisamente, la cláusula castellana cumple varias funciones al mismo tiempo y, por supuesto, esta idea es uno de los pilares de la Gramática Sistémica que, como el mismo Fawcett reconoce, le debemos a Halliday y ha dado lugar a la hipótesis del Principio Multifuncional del lenguaje.

Segundo, el Operador castellano siempre se amalgama con una de estas dos unidades: el Verbo Principal o el Auxiliar. Este último tiene precedencia, en caso de estar presente. El Verbo Principal es la opción por defecto. En alguna medida, todos los verbos castellanos se comportan como el verbo inglés be, que, según Fawcett (2008), es el único verbo que puede amalgamarse con el Operador. Esto es lo que ocurre en (35): 
(35) She's pretty.

$\{[S][M / O][C]\}$

Hemos visto que, en castellano, resulta plausible la hipótesis de que esa amalgama se da siempre. Nunca existe un Operador 'independiente', seguramente porque no forma parte en la determinación del Modo a nivel sintáctico. Los ejemplos (36) y (37) vuelven a mostrar casos de amalgamas.

(36) Ella estudia Filosofía.

$\{[\mathrm{S}][\mathrm{M} / \mathrm{O}][\mathrm{C}]\}$

(37) Ella había estudiado Filosofía.

$\{[S][X / O][M][C]\}$

En conclusión, lo que se denomina Operador puede identificarse perfectamente con la flexión, y es un elemento fundamental para la determinación del Modo de la cláusula, pero no desde el punto de vista de la disposición sintáctica (como en el inglés), sino desde la morfología.

Tercero, puede sugerirse algo así como un Principio de Participación para el Sujeto castellano: El Sujeto 'siempre' es un Rol Participante.

Cuarto, el Test del Sujeto en español debe tener en cuenta la concordancia entre el Sujeto y el Operador amalgamado con el Verbo Principal o el Auxiliar. Ahora bien, mientras que en inglés el Sujeto desempeña un papel clave en la expresión del significado interpersonal, no ocurre lo mismo en castellano. El Modo se manifiesta en la sintaxis inglesa por ejemplo por medio de la estructura S O, que significa 'dadora de información', o de la estructura O S, que significa ‘buscadora de polaridad'. Sobre la base de un análisis muy simple pudimos concluir que no es éste el caso para el español. Sin embargo, y aun cuando no contribuye fundamentalmente a la trama interpersonal del significado, el Sujeto español tiene algo que ver con ella. En primer lugar, el Sujeto expresa un significado gramaticalmente redundante, como réplica de la conjugación de la forma amalgamada $\mathrm{M} / \mathrm{O}$ o X/O. En efecto, el Sujeto español no es necesario para expresar el Modo ni la conjugación; sin embargo, cuando está explayado, sí participa (redundantemente) en la expresión del significado que ya está en la amalgama M/O o en la amalgama X/O. En segundo lugar, el Sujeto español también puede expresar un conjunto de significados comunicativamente pertinentes, comparables a los que expresa el Vocativo. Con respecto a este último punto, cabe señalar que ese significado puede ser también afectivo, una cuestión que podría dar lugar a más investigaciones sobre la función del Sujeto castellano.

Quinto, en inglés, la realización y la ubicación sintáctica del Sujeto se asocian directamente a la hebra interpersonal del significado. Por su parte, en español, la realización explícita del Sujeto se asocia (de una manera quizá más fuerte que en inglés) a la expresión del significado textual, puesto que su presencia sirve para destacar algún aspecto del mensaje. La de Sujeto Explícito es una opción vinculada a la hebra temática. Puede interpretarse que por medio de 
la realización del Sujeto Explícito se quiere expresar un significado pertinente o adicional. Al respecto, compárense las columnas de la Tabla 2: En la segunda, al haber optado por la regla de Sujeto Explícito, el hablante actualiza una instanciación por medio de la cual comunica que el Sujeto representa un elemento pertinente del mensaje. En este sentido, la Lingüística Sistémico-Funcional nos ayuda a mostrar por qué los ejemplos de la columna A en la Tabla 2 'no significan lo mismo' que los ejemplos de la columna B, aun cuando haya coincidencia desde el punto de vista de la TRANSITIVIDAD. En la Tabla 2 también se sugiere que los casos con Sujeto Tácito son 'no marcados' y que los de Sujeto Explícito son los casos 'marcados'.

Tabla 2. Una comparación de cláusulas ‘no marcadas' con otras ‘marcadas'.

\begin{tabular}{|c|c|}
\hline $\begin{array}{c}\text { Cláusula no marcada } \\
\text { (con Sujeto Tácito) }\end{array}$ & $\begin{array}{c}\text { Cláusula marcada } \\
\text { (con Sujeto Explícito) }\end{array}$ \\
\hline Está soleado & $\underline{\text { El día está soleado. }}$ \\
\hline Quiero té. & $\underline{\text { Yo quiero té. }}$ \\
\hline Estás loca. & $\underline{\text { Vos estás loca. }}$ \\
\hline ¿Me dan un certificado? & ¿Me dan ustedes un certificado? \\
\hline Que estudien en serio. & Que ellos estudien serio. \\
\hline ¡Salí de acá! & ¡Salí de acá vos! \\
\hline
\end{tabular}

Sexto, el rol de Sujeto como Tema es ampliamente compartido por muchas lenguas, entre las que se encuentran el inglés, el castellano y probablemente todas las lenguas germánicas y romances. En inglés, el Sujeto-Tema se realiza casi siempre de manera manifiesta, quizá por rol primordial que cumple al expresar el Modo. Existen varios tipos de cláusula donde el sujeto 'no está explayado pero es recuperable’ (Fawcett, 1999), por ejemplo, las ‘directivas no marcadas' como (38) o casos de Sujeto elidido como el de la segunda coordinada de (39).

(38) Do it!

(39) Eva had a bath and [Ø] came back home.

Digamos que en inglés todas las cláusulas (excepto las de Sujeto 'vacío' como (38) o (39)) tienen un Sujeto-Tema. El resultado es que el Sujeto-Tema aparece muy frecuentemente y es un tipo de Tema muy sencillo, no constituyéndose en una opción en la hebra del Modo, sino en la del TEMA. Por otra parte, en español resulta frecuente que el Sujeto esté Tácito y tampoco es extraño que haya oraciones 'sin Sujeto'. Al parecer, el Sujeto tiene una tendencia todavía más 
fuerte para expresar la hebra textual del significado. Así como el Sujeto es un constituyente decisivo para la expresión del Modo inglés, resulta clave para la expresión del Tema castellano. Los ejemplos de la Tabla 2 son ilustrativos al respecto. En castellano, la explicitud del Sujeto es una opción temática de importancia; se jerarquiza un elemento cuya expresión no es imprescindible en términos transitivos o impersonales.

Séptimo, ¿qué ocurre entonces con la expresión del Modo en castellano? Como ya se ha dicho, en la cláusula castellana se expresa el Modo por medio del Operador y la entonación (ver figuras 2 y 4). Los rasgos morfológicos de persona y número (que se amalgaman con el Verbo Principal o el Auxiliar) y la entonación, resultan suficientes para entender si una cláusula es 'dadora de información' como (40), ‘buscadora de información’ como (41), o ‘propuesta de acción’ como (42).

(40) Pateó Corti.

(41) ¿Pateó Corti?

(42) ¡Pateá, Corti!

Sin embargo, decir que el Modo se expresa mediante la morfología y la entonación es recién un punto de partida para futuras investigaciones que deberán caracterizar el complejo sistema de Modo en nuestra lengua.

Octavo, la forma que adopta el sistema léxico-gramatical de una lengua está determinada por las necesidades sociales y comunicativas que el lenguaje tiene que satisfacer. Todas las lenguas del mundo tendrían redes de rasgos semánticos cuyo fin es satisfacer esas necesidades. Sin embargo, el aspecto particular que toman las redes semánticas y las reglas de realización en una lengua puede variar sensiblemente con respecto a otras. Por ejemplo, el Modo inglés se realiza por medio de la sintaxis, i.e., por medio de la disposición sintáctica del Sujeto y el Operador. En el caso de nuestra lengua, el Modo se realiza principalmente a través de rasgos morfológicos y fonológicos tales como la conjugación verbal y los tipos de tono. En el marco general de un sistema lingüístico, unidades tales como el Sujeto y el Operador ingleses o la flexión verbal y la entonación españolas, sirven para expresar si el hablante se asigna a sí mismo el rol de 'dador de información', ‘buscador de información', 'promotor de una acción', etc. Así como la sintaxis inglesa parece haber evolucionado para expresar el Modo, es razonable sugerir que la morfología y la fonología no desempeñan en esta lengua un papel importante para ello. (Casos relativamente 'marginales' podrían ser ejemplos como You're coming over? o I am the one who should change?, con una 'entonación interrogativa'). Por otro lado, como en el español la fonología y la morfología son indispensables para la expresión del Modo, los rasgos sintácticos 
no cumplen en este caso ninguna función significativa. Pero en ambas lenguas existen unidades léxico-gramaticales (y fonológicas) que participan en la expresión del Modo y, por supuesto, en la expresión de otras hebras del significado.

Por último, las cuestiones aquí tratadas acaso tengan implicancias en lo referido al vínculo entre 'lenguaje y pensamiento'. Como la expresión del Modo en inglés se realiza manifiestamente por medio de la sintaxis, no es poco plausible sugerir que los lingüistas que son hablantes nativos del inglés se vieron predispuestos, por la estructura misma de la lengua inglesa, a percibir 'más fácilmente' la expresión del Modo (más fácilmente que los hablantes nativos de otras lenguas, como el castellano). Es probable que eso los haya llevado a vislumbrar la multifuncionalidad de la cláusula, unidad elemental de la léxico-gramática de toda lengua.

\section{REFERENCIAS BIBLIOGRÁFICAS}

Ball, F. \& Tucker, G. (2004). On the preferential co-occurence of Processes and Circumstantial Adjuncts: Some corpus evidence. En J. Foley (Ed.), Language, Education and Discourse (pp. 305-323). Londres: Continuum.

Banks, D. (2003). A note on modality in French, Word, 54(3), 325-334.

Barrenechea, A. \& Rosetti, M. (1986). Estudios de gramática estructural. Buenos Aires: Paidós.

Butler, C. (2003a). Structure and function: An introduction to three major structural-functional theories. I: Approaches to the simplex clause. Ámsterdam: John Benjamins.

Butler, C. (2003b). Structure and function: An introduction to three major structural-functional theories. II: From clause to discourse and beyond. Ámsterdam: John Benjamins.

Caffarel, A. (2004). Metafunctional profile of the grammar of French. En A. Caffarel, J. R. Martin \& C. Matthiessen (Eds.), Language Typology, A functional perspective (pp. 77137). Ámsterdam: John Benjamins.

Caffarel, A. (2006). A Systemic Functional Grammar of French. From grammar to discourse. Londres: Continuum.

Castel, V. (2007). Rule types in a Systemic Functional Grammar: An XML definition of the Cardiff Lexicogrammar Generator. En B. Leila \& T. Berber Sardinha (Eds.), Proceedings of the 33rd International Systemic Functional Congress (pp. 270-299). San Pablo: PUCSP.

Castel, V. (2008). A Systemic Functional micro-grammar of Spanish clitics. En C. Wu, C. Matthiessen \& M. Herke (Eds.), Proceedings of ISFC 2008: Voices around the world (pp. 7-12). ISFC: Sydney.

Danes, F. (1974). Papers on functional sentence perspective. Praga: Academia Checoslovaca de Ciencias. 
Fawcett, R. (1999). On the subject of the Subject in English: Two positions on its meaning (and on how to test for it). Functions of Language, 6(2), 243-273.

Fawcett, R. (2000a). A Theory of Syntax for Systemic Functional Linguistics. Ámsterdam: John Benjamins.

Fawcett, R. (2000b). In place of Halliday's 'verbal group'. Part 1: Evidence from the problems of Halliday's representations and the relative simplicity of the proposed alternative. Word, 51(2), 157-203.

Fawcett, R. (2000c). In place of Halliday's 'verbal group'. Part 2: Evidence from generation, semantics and interruptability'. Word, 51(3), 327-75.

Fawcett, R. (2007a). Auxiliary extensions: Six new elements for describing English. En R. Hasan, C. Matthiessen \& J. Webster (Eds.), Continuing Discourse on Language: A Functional Perspective (pp. 710-735). Londres: Equinox.

Fawcett, R. (2007b). Modelling "selection" between referents in the English nominal group: An essay in scientific inquiry in linguistics. En C. Butler, R. Hidalgo Downing \& J. Lavid (Eds.), Functional Perspectives on Grammar and Discourse: Papers In Honour of Angela Downing (pp. 165-204). Amsterdam: John Benjamins.

Fawcett, R. (2008). Invitation to Systemic Functional Linguistics. The Cardiff Grammar as an extension and simplification of Halliday's Systemic Functional Grammar. Beijing: Beijing University Press.

Fawcett, R. (en prensa) The many types of themes in English: Their syntax, semantics and discourse functions [en línea]. Disponible en: http: //www.isfla.org/Systemics/Print/ index.html

Gil, J. M. (2001). Introducción a las teorías lingüísticas del siglo XX. Santiago de Chile: Red Internacional del Libro.

Gouveia, C. \& Barbara, L. (2005). Tema e estrutura temática em portugués europeo e portugués brasileiro: Um estudo contrastivo das traduções portuguesa e brasileira de um original inglês. En M. do Oliveira, B. Hemais \& B. Gunnarson (Eds.), Comunicação, Cultura e Interação em Contextos Organizacionais (pp. 435-457). Río de Janeiro: Papel Virtual Editora.

Halliday, M. (1967a). Notes on transitivity and theme in English (I). Journal of Linguistics, 3(1), 37-81.

Halliday, M. (1967b). Notes on transitivity and theme in English (II). Journal of Linguistics, 3(2), 199-244.

Halliday, M. (1968). Notes on transitivity and theme in English (III). Journal of Linguistics, 4(1), 179-215.

Halliday, M. (1970). Language structure and language function. En J. Lyons (Comp.), New Horizons of Linguistics (pp. 140-165). Londres: Penguin. 
Halliday, M. (1978). El lenguaje como semiótica social. Bogotá: FCE.

Halliday, M. (1985). An introduction to Functional Grammar. Londres: Arnold.

Halliday, M. (1994). An introduction to Functional Grammar (segunda edición). Londres: Arnold.

Halliday, M. \& Matthiessen, C. (2004). An introduction to Functional Grammar (tercera edición). Londres: Arnold.

Huang, G. (1996). Experiential enhanced theme in English. En M. Berry, C. Butler, R. Fawcett \& G. Huang (Eds.), Meaning and Form: Systemic Functional Interpretations (Meaning and Choice in Language: Studies for Michael Halliday) (pp. 65-81). Norwood, NJ: Ablex.

Huang, G. (2002). Enhanced theme in English: Its structures and functions. Shangxi: Shangxi Educational Press.

Montemayor-Borsinger, A. (1998) Análisis de Tema-Rema y cadenas léxicas en libros de texto. Anales del Instituto de Lingüística de la Universidad Nacional de Cuyo, 18(21), 151174.

Kovacci, O. (1992). El comentario gramatical. Madrid: Arco.

Rossetti, M. \&. Zamudio, B. (1984). La gramática actual: Nuevas dimensiones. Buenos Aires: Plus Ultra.

Salvi, G. \& Vanelli, L. (2004). Nuova grammatica italiana. Bologna: Il Mulino.

Tench, P. (1996). The intonation systems of English. Londres: Cassell Academic.

Tucker, G. (1998). The lexicogrammar of adjectives: A Systemic Functional approach to Lexis. Londres: Cassell Academic. 